\title{
INCOMPLETE SELF-ORTHOGONAL LATIN SQUARES
}

\author{
KATHERINE HEINRICH and L. ZHU
}

(Received 14 May 1985)

Communicated by W. Wallis

\begin{abstract}
We show that for all $n \geqslant 3 k+1, n \neq 6$, there exists an incomplete self-orthogonal latin square of order $n$ with an empty order $k$ subarray, called an ISOLS $(n ; k)$, except perhaps when $(n ; k) \in\{(6 m$ $+i ; 2 m): i=2,6\}$.
\end{abstract}

1980 Mathematics subject classification (Amer. Math. Soc.): 05 B 15.

\section{Notation and basic constructions}

We study a particular family of incomplete orthogonal latin squares.

Let ILS $\left(n ; b_{1}, b_{2}, \ldots, b_{k}\right)$ be an order $n$ array $A$ with entries from an $n$-set $B$ defined as follows, where the $B_{i}, 1 \leqslant i \leqslant k$, are subsets of $B$ so that $\left|B_{i}\right|=b_{i}$, and where $B_{i} \cap B_{j}=\varnothing, 1 \leqslant i, j \leqslant k$.

(a) Each cell of $A$ is empty or contains an element of $B$;

(b) the subarrays indexed by $B_{i} \times B_{i}$ are empty; and

(c) the elements in row or column $b$ are exactly those of $B \backslash B_{i}$ if $b \in B_{i}$, and of $B$ otherwise.

Two ILS $\left(n ; b_{1}, b_{2}, \ldots, b_{k}\right)$ are orthogonal if, on superposition, all ordered pairs $(B \times B) \backslash \bigcup_{i=1}^{k}\left(B_{i} \times B_{i}\right)$ result. Denote two such squares by $\operatorname{IPOSL}\left(n ; b_{1}, b_{2}, \ldots, b_{k}\right)$. Similarly, $r-\operatorname{IPOLS}\left(n ; b_{1}, b_{2}, \ldots, b_{k}\right)$ denotes a set of $r \operatorname{ILS}\left(n ; b_{1}, b_{2}, \ldots, b_{k}\right)$ which are pairwise orthogonal. A pair of orthogonal $\operatorname{ILS}\left(n ; b_{1}, b_{2}, \ldots, b_{k}\right)$ in which one is the transpose of the other (a self-orthogonal array) will be denoted $\operatorname{ISOLS}\left(n ; b_{1}, b_{2}, \ldots, b_{k}\right)$. In the case when $b_{i}=0$ for each $i$ the arrays are all latin squares, and we denote them respectively by $\operatorname{LS}(n)$, $\operatorname{POLS}(n), r-\operatorname{POLS}(n)$ and $\operatorname{SOLS}(n)$.

(C) 1987 Australian Mathematical Society 0263-6115/87 \$A2.00+0.00 
We are interested in the existence of $\operatorname{ISOLS}(n ; k)$ and will show that, except for two infinite families, an ISOLS $(n ; k)$ exists if and only if $n \geqslant 3 k+1, n \neq 6$. The infinite families are ISOLS $(6 m+2 ; 2 m)$ and ISOLS $(6 m+6 ; 2 m)$. While many of the second family can be constructed, none of the first is known and, in particular, computer search verifies that there is no ISOLS $(8 ; 2)$.

This problem has been studied for quite some time. Simple counting shows that $n \geqslant 3 k+1$ is a necessary condition, and many $\operatorname{ISOLS}(3 k+1 ; k)$ were constructed by Parker [17] (notably an ISOLS(10;3)) and Hedayat [10]. Crampin and Hilton [5] showed that for every $k$ there exists an $n(k)$ such that, for all $n>n(k)$, an ISOLS $(n ; k)$ can be constructed. This was greatly improved by Drake and Lenz [9] who gave constructions for ISOLS $(n ; k)$ whenever $k \geqslant 304$ and $n \geqslant 4 k+3$.

We will use two types of construction: generalized product constructions and a starter-adder type construction. The product constructions are adaptions of the Wilson-type constructions given by Brouwer and van Rees [4], although they give them in terms of orthogonal arrays. It is assumed that the reader is familiar with the usual product constructions: direct and semi-direct product.

First we state results on the existence of special sets of orthogonal latin squares.

Lemma 1.1 (Lindner, Mullin, Stinson [16], Wang [19], Zhu [23]). For all $p$, $p \notin E=\{2,3,6,10,14,46,54,58,62,66,70\}$ there exists an $\operatorname{SOLS}(p)$ with a symmetric orthogonal mate.

LEMMA 1.2 (Wang [19]). For all even $p, p \notin F=E \cup\{78,82,98,102$, $118,142,174,194,202,214,230,258,278,282,394,398,402,422,1322\}$, there exists an $\operatorname{SOLS}(p)$ with a symmetric orthogonal mate which has only the entry 1 on the main diagonal.

Call the above SOLS $(p) P$ and the symmetric orthogonal mate $P_{1}$. Now, let $P_{0}$ denote the $\operatorname{LS}(p)$ defined by $e_{P_{0}}(s, t) \equiv t-s(\bmod p)$ and based on the elements $\{1,2, \ldots, p\}$, where $e_{X}(s, t)$ is the entry in cell $(s, t)$ of the array.

Lemma 1.3. For all $p, \operatorname{gcd}(p, 6)=1$, there exist orthogonal latin squares $P$ and $P_{1}$ of order $p$ so that each square is orthogonal to $P_{0}$.

Proof. Let $P$ be the SOLS $(p)$ with $e_{p}(s, t) \equiv 2 t-s(\bmod p)$, and let $P_{1}$ be defined by $\dot{e}_{p_{1}}(s, t) \equiv t+s(\bmod p)$.

LEMMA 1.4. For all odd prime powers $p, p \geqslant 5$, there exists a set of $(p-1)$ $\operatorname{POLS}(p)$ which consists of $P_{0}$, a symmetric square $P_{1}$ and $(p-3) / 2$ self-orthgonal squares $P, P_{2}, \ldots, P_{(p-3) / 2}$ and their transposes. 
Proof. This result comes immediately from the construction of a complete set of latin squares of order $p$. (See, for example, [6, pp. 160-169].)

Note that if $n=p_{1}^{e_{1}} p_{2}^{e_{2}} \cdots p_{k}^{e_{k}}$, where the $p_{i}$ are distinct odd primes, and if $r=p_{1}^{e_{1}}<p_{2}^{e_{2}}<\cdots<p_{k}^{e_{k}}$, then there are (by direct product) squares $P, P_{0}, P_{1}$, $P_{2}, \ldots, P_{(r-3) / 2}$ of order $n$.

The idea of the product construction is to take the square $P$ and replace each cell by a $q \times q$ array; this array will in general either be one of a $\operatorname{POLS}(q)$ or, combined with additional rows and columns added to $P$, one of an IPOLS $(q+$ $x ; x)$.

If we have a symmetric transversal in $P$ (determined say by the entry 1 in $P_{1}$, or $p$ in $P_{0}$ if $p$ is odd), then we add $a_{11}$ new rows and columns to $P$. Replace the cells $(i, j)$ and $(j, i), i \neq j$, of $P$ determined by the transversal with one of an $\operatorname{IPOLS}\left(q+a_{11} ; a_{11}\right)$ and its transpose, respectively; any cell $(i, i)$ of the transversal with an ISOLS $\left(q+a_{11} ; a_{11}\right)$; and all other cells with either a POLS $(q)$ and its transpose or an $\operatorname{SOLS}(q)$ (if the cell is on the main diagonal). This yields an $\operatorname{IPOLS}\left(p q+a_{11} ; a_{11}\right)$.

If we have a pair of symmetrically placed transversals $T_{1}$ and $T_{p-1}$ in $P$, determined by say 1 and $p-1$ in $P_{0}$, then we add $2 a_{10}$ rows and columns to $P$. If $(i, j)$ is a cell of $T_{1}$, then it is replaced by one of an IPOLS $\left(q+a_{10} ; a_{10}\right)$ using columns $p q+1, \ldots, p q+a_{10}$ and rows $p q+a_{10}+1, \ldots, p q+2 a_{10}$, and cell $(j, i)$ is then replaced by the transpose of the other using columns $p q+a_{10}+$ $1, \ldots, p q+2 a_{10}$ and rows $p q+1, \ldots, p q+{ }_{10}$. Other cells are replaced by $\operatorname{POLS}(q)$ and its transpose or by an SOLS $(q)$. This yields an ISOLS $(p q+$ $\left.2 a_{10} ; 2 a_{10}\right)$.

Finally, if we have a pair of symmetrically placed transversals $T$ and $S$ in $P$, determined say by 1 in $P_{j}$ and $P_{j}^{T}$, then we add $2 a_{i j}$ new rows and columns to $P$. (Note that $T$ and $S$ intersect in cell $(1,1)$.) Now, as before, if $(i, j)$ is a cell of $T$, replace it by one of a POLS $\left(q+a_{j} ; a_{j}\right)$ using columns $p q+1, \ldots, p q+a_{j}$ and rows $p q+a_{j}+1, \ldots, p q+2 a_{j}$. At the same time $(j, i)$ is replaced by the transpose of the other of the IPOLS $\left(q+a_{j} ; a_{j}\right)$ using columns $p q+a_{j}+$ $1, \ldots, p q+2 a_{j}$ and rows $p q+1, \ldots, p q+a_{j}$. Other cells (except $(1,1)$ ) are replaced by one of a POLS $(q)$ and its transpose or an $\operatorname{SOLS}(q)$. Now using cell $(1,1)$ we get an ISOLS $\left(p q+2 a_{j} ; q+2 a_{j}\right)$.

In many cases these constructions can be combined, and this is the substance of the next lemmas. To begin with, let $a_{j} \in Z^{+} \cup\{0\}$ be associated with the entry 1 of $P_{j}$ and $P_{j}^{T}, 2 \leqslant j \leqslant r$; that is, we will add $a_{j}$ to each of these transversals in $P$. Let $a_{k, 0}=a_{p-k, 0} \in Z^{+} \cup\{0\}, 1 \leqslant k \leqslant(p-1) / 2$ ( $p$ is odd), be associated with the entries $k$ and $p-k$ of $P_{0}$; let $a_{p 0}$ be associated with $p$ on the main diagonal. Let $a_{k 1} \in Z^{+} \cup\{0\}, 1 \leqslant k \leqslant p$, be associated with $k$ in $P_{1}$. Then let $a_{0}=$ $\sum_{k=1}^{p} a_{k 0}, \quad a_{1}=\sum_{k-1}^{p} a_{k 1}$ and $a=a_{0}+a_{1}+2 \sum_{k=2}^{r} a_{k}$. Finally, let $A_{0}=$ $\left\{a_{10}, a_{20}, \ldots, a_{(p-1) / 2,0}, a_{p 0}\right\}$ and $A_{1}=\left\{a_{11}, a_{21}, \ldots, a_{p 1}\right\}$. 
Most constructions will be given without proof. Also note that, although the premises of the lemma suppose the existence of certain squares, the constructions are still valid if we do not have $P_{1}\left(\right.$ all $\left.a_{j 1}=0\right), P_{0}\left(\right.$ all $\left.a_{j 0}=0\right)$ or $P_{t}\left(a_{t}=0\right)$. So when the lemmas are later referred to, we may not have all the squares. Moreover, even without the square $P_{0}$ we can still use the transversal on the main diagonal of $P$.

LEMma 1.5. Suppose we have squares $P, P_{1}$ and $P_{0}$ of order $p$. Then, if there are $\operatorname{ISOLS}\left(q+a_{p 0}+a_{j 1} ; a_{p 0}, a_{j 1}\right)$ and $\operatorname{IPOLS}\left(q+a_{i 0}+a_{j 1} ; a_{i 0}, a_{j 1}\right)$, there is an $\operatorname{ISOLS}\left(p q+a ; a_{0}, a_{1}\right)$. Hence if an $\operatorname{SOLS}\left(a_{0}\right)\left(\operatorname{SOLS}\left(a_{1}\right)\right)$ exists, then we have an $\operatorname{ISOLS}\left(p q+a ; a_{1}\right)\left(\operatorname{ISOLS}\left(p q+a ; a_{0}\right)\right)$.

Proof. Add $a_{0}+a_{1}$ rows and columns to $P$ as in the earlier discussion. If $(s, t), s \neq t$, is a cell of $P$, and if $e_{P_{1}}:(s, t)=j, e_{P_{0}}(s, t)=i$, then by using the appropriate rows and columns (that is, we use the same $a_{j 1}$ rows as columns, but we use the first $a_{i 0}$ of $2 a_{i 0}$ rows and the second $a_{i 0}$ of $2 a_{i 0}$ columns), replace this cell by one of an IPOLS $\left(q+a_{i 0}+a_{j 1} ; a_{i 0}, a_{j 1}\right)$ and replace $(t, s)$ by the transpose of its mate. On the main diagonal cells use the $\operatorname{ISOLS}\left(q+a_{p 0}+a_{j 1} ; a_{p 0}, a_{j 1}\right)$ (with the same $a_{p 0}$ rows and columns, and the same $a_{j 1}$ rows and columns).

LEMMA 1.6. If we have squares $P, P_{1}$ and $P_{0}$ of order $p$, and if we have $\operatorname{ISOLS}\left(q+a_{p 0}+a_{j 1} ; x, a_{p 0}, a_{j 1}\right), \operatorname{IPOLS}\left(q+a_{i 0}+a_{j 1} ; x, a_{i 0}, a_{j 1}\right), \operatorname{ISOLS}\left(a_{0}\right)$ and $\operatorname{ISOLS}\left(a_{1}\right)$, then we have an $\operatorname{ISOLS}(p q+a ; p x)$. Moreover, if we also have an ISOLS $\left(q+a_{i 0}+a_{j 1} ; x, a_{i 0}, a_{j 1}\right)$, then we have ISOLS $(p q+$ $\left.a ; p^{q-x}, p x, a_{0}, a_{1}\right)$, where $p^{q-x}$ indicates $q-x$ order $p$ arrays.

Note that in the constructions of Lemmas 1.5 and 1.6 there are also other subsquares. For example, if in Lemma $1.5, a_{p 0}=a_{j 1}=0$ for some $j$, where $e_{P_{1}}(s, s)=j$, and we have both an $\operatorname{SOLS}\left(a_{0}\right)$ and an $\operatorname{SOLS}\left(a_{1}\right)$, then we get an ISOLS $(p q+a ; q)$.

Lemma 1.7. Suppose we have squares $P, P_{2}, \ldots, P_{r}$ of order $p$. Then if we have $\operatorname{IPOLS}\left(q+a_{j} ; q_{j}\right), 2 \leqslant j \leqslant r$, and $\operatorname{IPOLS}(q)$, we can construct an ISOLS( $p q+$ $a ; q+a)$.

Proof. Simply add rows and columns and extend using each of $P_{j}$ and $P_{j}^{T}$ in turn. Ignore the cell $(1,1)$ of $P$, which is contained in all transversals. Leaving this cell and the corresponding rows and columns empty yields the ISOLS( $p q+a ; q$ $+a)$.

We can now combine the constructions of Lemmas 1.5 and 1.7. 
Lemma 1.8. Given squares $P, P_{0}, P_{1}, P_{2}, \ldots, P_{r}$ of order $p$, given IPOLS $\left(a+a_{i 0}\right.$ $\left.+a_{j 1}+a_{k} ; a_{i 0}, a_{j 1}, a_{k}\right)$, ISOLS $\left(q+a_{p 0}+a_{j 1} ; a_{p 0}, a_{j 1}\right)$, ISOLS $\left(a_{0} ; a_{p 0}\right)$ and ISOLS $\left(a_{1} ; a_{11}\right)$, we have an ISOLS $\left(p q+a ; q+a_{p 0}+a_{11}+2\left(a_{2}+\cdots+a_{r}\right)\right)$.

Proof. This is apparent upon noting that the empty subarray comes from the cell $(1,1)$ in $P$ through which most transversals pass (see Figure 1.1). Again, this lemma allows for many more subsquares, as can be seen from Figure 1.1 below. In particular, note that if we have no $P_{2}, \ldots, P_{r}$, then we get ISOLS $(p q+a ; q+$ $a_{p 0}+a_{11}$.

LeMma 1.9. Given squares $P, P_{0}, P_{1}, P_{2}, \ldots, P_{r}$ of order $p$, given IPOLS $\left(q+a_{i 0}\right.$ $\left.+a_{j 1}+a_{k} ; x, a_{i 0}, a_{j 1}, a_{k}\right), \operatorname{ISOLS}\left(q+a_{p 0}, a_{j 1} ; x, a_{p 0}, a_{j 1}\right), \operatorname{ISOLS}\left(a_{0} ; a_{p 0}\right)$, $\operatorname{ISOLS}\left(a_{1} ; a_{11}\right)$ and $\operatorname{ISOLS}\left(q+a_{p 0}+a_{11}+2\left(a_{2}+\cdots+a_{r}\right) ; x\right)$, we have an ISOLS $(p q+a ; p x)$.

Finally, we note a trivial result.

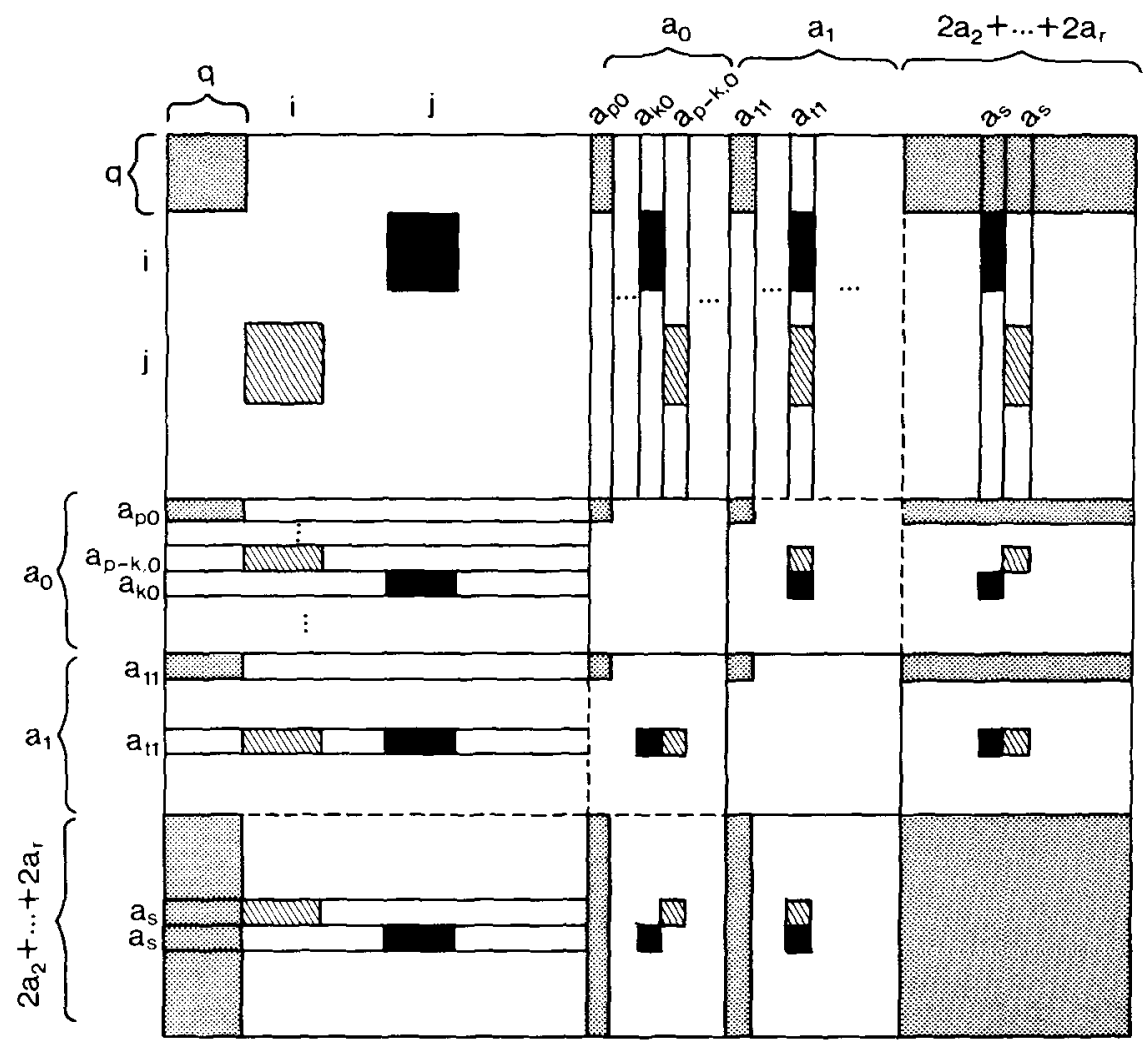

FIGURE 1.1 
LEMMA 1.10. Given an $\operatorname{ISOLS}(a ; b)$ and an $\operatorname{ISOLS}(b ; y)$, we have an $\operatorname{ISOLS}(a ; y)$.

Special cases of the next lemma have been given by Dinitz and Stinson [7].

LEMMA 1.11. Given an ISOLS $\left(n ; b_{1}, b_{2}, \ldots, b_{k}\right)$, where $n=\sum_{i=1}^{k} b_{i}$, and an $\operatorname{ISOLS}\left(b_{i}+x ; x\right)$ for each $i \neq j$, we have an $\operatorname{ISOLS}\left(n+x ; b_{j}+x\right)$.

Our last technique is a starter-adder type construction. This idea has been described by several authors including Horton [15], Hedayat and Seiden [11], and Zhu [20]. The plan is to construct an $\operatorname{ISOLS}(n ; k) A$ from its first row (given by $\mathbf{e}=\left(e_{A}(1,1), \ldots, e_{A}(1, n-k)\right)$ and $\left.\mathbf{f}=\left(e_{A}(1, n-k+1), \ldots, e_{A}(1, n)\right)\right)$ and from the last $k$ entries of the first column (given by $\mathrm{g}=\left(e_{A}(n-k+\right.$ $\left.\left.1,1), \ldots, e_{A}(n, 1)\right)\right)$. The entries of the array are $\{1,2, \ldots, n-k\} \cup X$, where $X=\left\{x_{1}, x_{2}, \ldots, x_{k}\right\}$. The arrary is constructed modulo $n-k$ using a fixed $d \in\{1,2, \ldots, n-k\}$, where the $x_{i}$ act as "infinity" elements according to the following rules.

$$
\begin{aligned}
& e_{A}(s+1, t+1)=e_{A}(s, t) \text { if } e_{A}(s, t)=x_{i} \\
& \left.e_{A}(s+1, t+1) \equiv e_{A}(s, t)+d(\bmod n-k) \text { otherwise }\right\} \text {, } \\
& 1 \leqslant s, t \leqslant n-k,
\end{aligned}
$$

(b) $e_{A}(s+1, n-k+t) \equiv e_{A}(s, n-k+t)+d(\bmod n-k)$,

$$
1 \leqslant t \leqslant k, 1 \leqslant s \leqslant n-k \text {, }
$$

(c) $e_{A}(n-k+t, s+1) \equiv e_{A}(n-k+t, s)+d(\bmod n-k)$,

$$
1 \leqslant t \leqslant k, 1 \leqslant s \leqslant n-k .
$$

Note that in case (a) all cell labels are determined modulo $n-k$, but in cases (b) and (c) this applies only to the row and column labels respectively.

It is not difficult to determine the conditions that $\mathrm{e}, \mathrm{f}, \mathrm{g}$ and $d$ must satisfy, but we shall not concern ourselves with that. When needed, the value of $d$ and of the vectors $\mathbf{e}, \mathbf{f}$ and $g$ will be given. Simple calculations verify that they work. For example, an ISOLS $(20 ; 3)$ can be constructed from

$$
\begin{aligned}
& \mathbf{e}=\left(1,14,10,6,2, x_{1}, 11,7,3,16,12,8,4,17,13, x_{2}, x_{3}\right), \\
& \mathbf{f}=(5,9,15), \mathrm{g}=(13,2,3) \text { and } d=14 .
\end{aligned}
$$

\section{Main result}

We shall now apply the constructions described earlier to construct ISOLS $(n ; k), n \geqslant 3 k+1$. Throughout, $x, y, a_{i 0}, a_{i 1}$ and $a_{j}$ are given only when they are non-zero. The entries of $A_{0}$ and $A_{1}$ will be given, and, although it will 
not be indicated to which non-zero $a_{i 0}$ or $a_{i 1}$ we refer, it is always easy to decide. Also, as mentioned earlier, we abbreviate notation by writing $i^{j}$ when $i$ occurs $j$ times in a set.

The following results are crucial to the constructions.

THEOREM 2.1. For all $n \geqslant 3 k,(n ; k) \neq(6 ; 1)$, there exists an IPOLS $(n ; k)$.

The proof is given in a series of papers $([14,18,21,22])$, but can be found in its entirety in [13].

COROLlary 2.2. For all $n \geqslant 3 k+1$ with $(n ; k) \neq(6 ; 1)$, there is an $\operatorname{IPOLS}(n ; 1, k)$.

Theorem 2.3 (Brayton, Coppersmith and Hoffman [3]). For all $n \neq 2,3,6$, there exists an $\operatorname{SOLS}(n)$.

COROLlaRY 2.4. For all $n \neq 2,3,6$, there exists $a$ POLS $(n)$.

Corollary 2.5. For all $n \neq 2,3,6$, there exists an $\operatorname{ISOLS}\left(n ; 1^{r}\right), 1 \leqslant r \leqslant n$, (and hence an $\operatorname{IPOLS}\left(n ; 1^{r}\right)$ ).

LEMMA 2.6 (Heinrich [12]). There exists an ISOLS( $3 k+1 ; k)$ for all $k$.

Proof. Apply the starter-adder technique with

$$
\begin{aligned}
& \mathbf{e}=\left(1, x_{1}, x_{2}, \ldots, x_{k}, 2,4,6, \ldots, 2 k\right), \quad \mathbf{f}=(3,5,7, \ldots, 2 k+1), \\
& \mathbf{g}=(2,3,4, \ldots, k+1) \text { and } d=1
\end{aligned}
$$

LEMMA 2.7. There exists an ISOLS $(n ; 2)$ when $7 \leqslant n \leqslant 50$ and $n \neq 8$.

Proof. For $9 \leqslant n \leqslant 21, n \neq 18$, vectors for the starter adder technique are given in Table 2.1. (The case $n=7$ was given in Lemma 2.6.)

When $n=25$, apply Lemmas 1.8 and 1.10 with $p=7, q=3, a_{p 0}=a_{11}=a_{2}$ $=1$ and $y=2$. For $n=31,32$, apply Lemmas 1.9 and 1.10 with $p=7, q=4$, $x=1, y=2$ and, respectively, $a_{p 0}=a_{2}=1$ and $a_{p 0}=a_{11}=a_{2}=1$. The remaining orders $n$ are given in Table 2.2. 
TABLE 2.1

$\begin{array}{rllll}n & d & \mathbf{l} & \mathbf{f} & \mathbf{g} \\ 9 & 1 & \left(1, x_{1}, x_{2}, 5,7,2,6\right) & (3,4) & (6,5) \\ 10 & 1 & \left(1,3, x_{1}, 6,4, x_{2}, 2,5\right) & (7,8) & (5,7) \\ 11 & 1 & \left(1,3,5,7,9,2,6, x_{1}, x_{2}\right) & (4,8) & (8,7) \\ 12 & 1 & \left(1,4, x_{1}, 8, x_{2}, 5,3,6,10,7\right) & (2,9) & (4,6) \\ 13 & 1 & \left(1,4,8,3,6,10,2,11, x_{1}, x_{2}, 9\right) & (5,7) & (9,8) \\ 14 & 1 & \left(1,3,8,10, x_{1}, x_{2}, 6,11,5,12,9,4\right) & (2,7) & (10,8) \\ 15 & 1 & \left(1, x_{1}, x_{2}, 5,8,11,3,2,13,7,6,10,12\right) & (4,9) & (3,7) \\ 16 & 1 & \left(1,14,10,5,13,3,12,7,11, x_{1}, x_{2}, 4,2,9\right) & (6,8) & (5,11) \\ 17 & 1 & \left(1,3,8,6,11,14,2,5, x_{1}, x_{2}, 10,15,7,12,4\right) & (9,13) & (12,8) \\ 19 & 5 & \left(1,5,9,13, x_{1}, 4,8,12,16,3,7,11,15,2,6,10, x_{2}\right) & (14,17) & (2,14) \\ 20 & 1 & \left(1, x_{1}, x_{2}, 2,6,12,16,18,13,9,8,5,15,17,4,3,11,14\right) & (7,10) & (14,9) \\ 21 & 1 & \left(1, x_{1}, x_{2}, 2,4,7,16,15,19,13,6,14,9,18,8,5,3,12,11\right) & (10,17) & (17,7)\end{array}$

TABLE 2.2

\begin{tabular}{llrlll}
$n$ & $p$ & $q$ & $A_{0}$ & $A_{1}$ & Lemma(s) \\
\hline 18 & 4 & 4 & - & $\left\{1^{2}\right\}$ & 1.5 \\
22 & 5 & 4 & $\left\{1^{2}\right\}$ & - & 1.5 \\
23 & 7 & 3 & $\{1\}$ & $\{1\}$ & 1.6 and $1.10 x=1 \quad y=2$ \\
24 & 7 & 3 & $\{1\}$ & $\left\{1^{2}\right\}$ & 1.5 \\
26 & 7 & 3 & $\left\{1^{5}\right\}$ & - & 1.6 and $1.10 x=1 \quad y=2$ \\
27 & 7 & 3 & $\left\{1^{5}\right\}$ & $\{1\}$ & 1.6 and $1.10 x=1 y=2$ \\
28 & 7 & 4 & - & - & 1.6 and $1.10 x=1 \quad y=2$ \\
29 & 7 & 4 & $\{1\}$ & - & 1.6 and $1.10 x=1 y=2$ \\
30 & 7 & 4 & $\left\{1^{2}\right\}$ & - & 1.5 \\
33 & 7 & 4 & $\left\{1^{5}\right\}$ & - & 1.6 and $1.10 x=1 \quad y=2$ \\
34 & 4 & 8 & - & $\{2\}$ & 1.5 \\
35 & 7 & 5 & - & - & 1.6 and $1.10 x=1 \quad y=2$ \\
36 & 9 & 4 & - & - & 1.6 and $1.10 x=1 y=2$ \\
37 & 9 & 4 & $\{1\}$ & - & 1.6 and $1.10 x=1 y=2$ \\
38 & 9 & 4 & $\left\{1^{2}\right\}$ & - & 1.5 \\
39 & 5 & 7 & $\left\{1^{4}\right\}$ & - & 1.8 and $1.10 y=2$ \\
40 & 4 & 10 & - & - & 1.8 and $1.10 y=2$ \\
41 & 4 & 10 & - & $\{1\}$ & 1.8 and $1.10 y=2$ \\
42 & 4 & 10 & - & $\{2\}$ & 1.5 \\
43 & 5 & 7 & $\left\{2^{4}\right\}$ & - & 1.8 and $1.10 y=2$ \\
44 & 4 & 11 & - & - & 1.8 and $1.10 y=2$ \\
45 & 5 & 9 & - & - & 1.8 and $1.10 y=2$ \\
46 & 5 & 9 & - & $\{1\}$ & 1.8 and $1.10 y=2$ \\
47 & 5 & 9 & $\{2\}$ & - & 1.5 \\
48 & 4 & 12 & - & - & 1.8 and $1.10 y=2$ \\
49 & 4 & 12 & - & $\{1\}$ & 1.8 and $1.10 y=2$ \\
50 & 4 & 12 & - & $\{2\}$ & 1.5 \\
& & & & &
\end{tabular}

THEOREM 2.8. There exists an ISOLS( $n ; 2)$ for all $n \geqslant 7$ and $n \neq 8$.

Proof. By Lemma 2.7 we need only consider the case $n \geqslant 51$, and the proof is given in Table 2.3, where, in each case, $p=7$ and $q=k(k \geqslant 7)$. 
TABLE 2.3

\begin{tabular}{llll}
$n-2$ & $A_{0}$ & \multicolumn{1}{c}{$A_{1}$} & \multicolumn{1}{c}{ Lemma(s) } \\
\hline $7 k$ & $\left\{1^{2}\right\}$ & - & 1.5 \\
$7 k+1$ & $\left\{1^{2}\right\}$ & $\{1\}$ & 1.5 \\
$7 k+2$ & $\left\{1^{4}\right\}$ & - & 1.6 and $1.10 x=1 y=2$ \\
$7 k+3$ & $\left\{1^{5}\right\}$ & $\{1\}$ & 1.6 and $1.10 x=1 y=2$ \\
$7 k+4$ & $\left\{1^{2}\right\}$ & $\left\{1^{4}\right\}$ & 1.5 \\
$7 k+5$ & $\left\{1^{2}\right\}$ & $\left\{1^{5}\right\}$ & 1.5 \\
$7 k+6$ & $\left\{1^{4}\right\}$ & $\left\{1^{4}\right\}$ & 1.6 and $1.10 x=1 y=2$
\end{tabular}

We shall now construct ISOLS $(n ; k)$ for all $n \geqslant 3 k+1$ and $k \geqslant 7$, except when $k=2 m$ and $n=3 k+2$ or $3 k+6$. This leaves only finitely many values of $n$ for each $k, k \in\{3,4,5,6\}$ to be considered.

THEOREM 2.9. If $n=m k+\varepsilon, 0 \leqslant \varepsilon<k, k \geqslant 7, m \geqslant 7$, then there is an $\operatorname{ISOLS}(n ; k)$.

Proof. The case $\varepsilon=0$ is immediate by product.

First we consider the cases $k \notin E$.

If $\varepsilon \neq 2,3$, or 6 , apply Lemma 1.6 with $p=k, q=m, x=1$ and $A_{1}=\left\{1^{\varepsilon}\right\}$.

If $\varepsilon=2,3$ or 6 , use Lemma 1.6 with $p=k, q=m-1, x=1$ and $A_{1}=$ $\left\{1^{k-\varepsilon}, 2^{\varepsilon}\right\}$. If $k$ is even, there are at least $k / 2$ symmetric transversals (determined from $P_{1}$ ) which contain no cells of the main diagonal of $P$, and we can thus avoid the need for an ISOLS $(m+1 ; 2)$. But for odd $k$ this is not possible unless $m \neq 7$. When $m=7$ and $\varepsilon=2,3$, put $p=k, q=7, x=1, a_{p 0}=\varepsilon$ and apply Lemma 1.9; and when $\varepsilon=6$, repeat but with $a_{p 0}=a_{11}=3$.

Now, if $k \in E$, then $k \geqslant 10$ and $k-1 \notin F$. We consider separately the cases $m$ odd and $m$ even. Let $m=2 h+1$. Applying Lemma 1.6 with $p=k-1$, $q=m, x=1$ and $A_{1}=\left\{1^{\varepsilon}, h^{2}\right\}$, provided that $\varepsilon+2 \leqslant k-1$, in conjunction with Corollary 2.5 and Lemma 2.6, we obtain an ISOLS $(k m+\varepsilon-1$; $\left.(k-1)^{m}, m+\varepsilon-1\right)$. Now Lemma 1.11, applied to this with $x=1$, gives an ISOLS $(k m+\varepsilon ; k)$. However, we still have $\varepsilon \in\{k-2, k-1\}$ to consider. In these cases we repeat the above argument, but with $A_{1}=\left\{1^{k-4}, 2, h^{2}\right\}$ and $A_{1}=\left\{1^{k-5}, 2^{2}, h^{2}\right\}$, respectively.

Now let $m=2 h$. Assuming that $\varepsilon \leqslant k-6$, apply Lemma 1.6 (also using Corollary 2.5, Lemma 2.6 and Theorem 2.8) with $p=k-1, q=2 h-1, x=1$ and $A_{1}=\left\{2^{(k+\varepsilon) / 2},(h-1)^{2}\right\}$ if $\varepsilon$ is even, and $A_{1}=\left\{1,2^{(k+\varepsilon-1) / 2},(h-1)^{2}\right\}$ if $\varepsilon$ is odd. This can be seen to yield an ISOLS $\left(k m+\varepsilon-1 ;(k-1)^{m-1}, m+k+\varepsilon\right.$ $-2)$, and so by Lemma 1.11 with $x=1$, an $\operatorname{ISOLS}(k m+\varepsilon ; k)$. 
We still have to consider the cases $n=k m+(k-\delta), m$ even, $1 \leqslant \delta \leqslant 5$. These are all covered by Lemma 1.6 with $p=k / 2$ (which is odd), $q=2 m+1$, $x=2, a_{p 0}=1$ and $A_{1}=\left\{1^{(k / 2-\delta-1)}\right\}$, where $k / 2-\delta-1 \geqslant 0$ and $k / 2-\delta-$ $1 \neq 2,3,6$. This leaves the cases $k=10$ and $\delta=1,2,5$; and $k=14, \delta=3,4$.

For $k=10$ and $\delta=1,5$, apply Lemma 1.6 with $p=5, q=2 m+1, x=2$ and $A_{0}=\left\{1^{4}\right\}, A_{0}$ empty, respectively. For $\delta=2$, apply Lemma 1.8 with $p=m-1, q=10$ and $A_{1}=\left\{2^{9}\right\}$, provided that $m \geqslant 11$. When $m=8$, put $p=8, q=10$ and $A_{1}=\left\{2^{4}\right\}$ in Lemma 1.8 ; and when $m=10$, put $p=7$, $q=14$ and $A_{0}=\left\{2^{5}\right\}$ in Lemma 1.5 .

Finally, for $k=14$ and $\delta=3$, apply Lemma 1.6 with $p=7, q=2 m+1$, $x=2$ and $A_{0}=\left\{1^{4}\right\}$; and for $k=14$ and $\delta=4$, apply Lemma 1.6 with $p=7$, $q=2 m, x=2$ and $A_{0}=A_{1}=\left\{1^{5}\right\}$.

THEOREM 2.10. If $n=5 k+\varepsilon, 0 \leqslant \varepsilon<2 k, k \geqslant 7$, then there is an $\operatorname{ISOLS}(n ; k)$.

Proof. Again, the case $\varepsilon=0$ is immediate by product.

Suppose $\varepsilon$ is odd and $\varepsilon \neq 3,7$. Apply Lemma 1.8 with $p=5, q=k$ and $A_{0}=\left\{[\varepsilon / 2]^{2}\right\}, A_{1}=\{1\}$ if $0<\varepsilon \leqslant k ; A_{0}=\left\{[k / 2]^{2},[(\varepsilon-k) / 2]^{2}\right\}, A_{1}=\{1\}$ if $k<\varepsilon<2 k$. If $\varepsilon$ is even and $\varepsilon \neq 2$, repeat the procedure but with $A_{1}$ empty and, if $k<\varepsilon<2 k, A_{0}=\left\{[k / 2]^{2},[(\varepsilon-k+1) / 2]^{2}\right\}$. Should $\varepsilon \in\{2,3,7\}$, use Lemma 1.8 with $p=5, q=k-1$ and $A_{0}=\left\{1^{3}, 2^{2}\right\}, A_{0}=\left\{1^{3}, 2^{2}\right\}$ and $A_{1}=$ $\{1\}$, and $A_{0}=\left\{1,2^{2}, 3^{2}\right\}$ and $A_{1}=\{1\}$, respectively.

THEOREM 2.11. If $n=4 k+\varepsilon, 0 \leqslant \varepsilon<k, k \geqslant 7$, then there is an $\operatorname{ISOLS}(n ; k)$.

Proof. When $\varepsilon=0$, product yields the result. If $\varepsilon \neq 2,3,6$, apply Lemma 1.8 with $p=4, q=k, A_{1}=\left\{[\varepsilon / 2]^{2}\right\}$ if $\varepsilon$ is even, and $A_{1}=\left\{[\varepsilon / 2]^{2}, 1\right\}$ if $\varepsilon$ is odd. For $\varepsilon=3,6$, apply Lemma 1.8 with $p=4, q=k-1$ and $A_{1}=$ $\left\{1,[(\varepsilon+2) / 2]^{3}\right\}$. Finally, we consider the case $\varepsilon=2$. Provided that $k \neq 7,8$, the same lemma, but with $p=4, q=k-2$ and $A_{1}=\left\{2^{2}, 3^{2}\right\}$, will work. An ISOLS(30;7) comes from Lemma 1.6 with $p=7, q=3, x=1, A_{0}=\left\{1^{5}\right\}$ and $A_{1}=\left\{1^{4}\right\}$. The ISOLS $(34 ; 8)$ found by Wang [19] is given by $d=1$ and the vectors $\mathbf{e}=\left(1,26,24,23,22,19,18,16,21,6,17,13,15,2,20,25, x_{1}, 12\right.$, $\left.x_{2}, 10, x_{3}, x_{4}, x_{5}, x_{6}, x_{7}, x_{8}\right), \quad \mathrm{f}=(14,8,5,3,4,7,11,9)$ and $\mathrm{g}=$ $(4,16,19,24,8,26,5,11)$.

The final case $n=3 k+\varepsilon, 1 \leqslant \varepsilon<k$, is more difficult and will be done in a series of lemmas.

LEMMA 2.12. There exists an ISOLS $(3 k+3 ; k)$ for $k \geqslant 3$. 
Proof. Consider the residue class of $k$ modulo 4. Write $k$ as $4 t+8,4 t+9$, $4 t+6$ and $4 t+3, t \geqslant 0$. The "starter-adder" technique will be used. In all four cases $d=1$ and $\mathrm{e}=\left(1, x_{1}, x_{2}, \ldots, x_{k}, k+3, k+2, \ldots, 2\right), \mathrm{f}=(k+4, k+$ $5, \ldots, 2 k+3)$, and the first $4 t$ positions of $g$ are given by $g^{\prime}=(8,6,4,10, \ldots, 8 j$ $+8,8 j+6,8 j+4,8 j+10, \ldots, 8 t, 8 t-2,8 t-4,8 t+2)$. Respectively, the last $8,9,6,3$ positions of $\mathbf{g}$ are given by $\mathbf{g}^{\prime \prime}$, where

$$
\begin{aligned}
& \mathbf{g}^{\prime \prime}=(8 t+6,8 t+10,8 t+4,8 t+8,8 t+12,8 t+18,8 t+16,8 t+14), \\
& \mathbf{g}^{\prime \prime}=(8 t+8,8 t+6,8 t+4,8 t+12,8 t+10,8 t+16,8 t+20,8 t+14,8 t+18), \\
& \mathbf{g}^{\prime \prime}=(8 t+8,8 t+6,8 t+4,8 t+14,8 t+10,8 t+12), \text { and } \\
& \mathbf{g}^{\prime \prime}=(8 t+8,8 t+6,8 t+4) .
\end{aligned}
$$

The cases $k=4,5$ remain. When $k=5, g=(6,10,4,8)$, and when $k=5$, $\mathrm{g}=(10,12,8,6,4)$.

LEMMA 2.13 (Heinrich [12]). There exists an ISOLS( $3 k+2 ; k)$ for $k \geqslant 3$ and $k$ odd.

Proof. Again, we simply give $d$ and vectors e, f and g. Let $k=2 m+1$ and put $d=1$. Then

$$
\begin{aligned}
& \mathbf{e}=\left(1, x_{1}, 2, x_{2}, \ldots, m+1, x_{m+1}, 4 m+4, m+2, x_{m+2},\right. \\
& \left.m+3, x_{m+3}, \ldots, 2 m+2\right), \\
& \mathbf{f}=(3 m+3,2 m+3,4 m+3,2 m+4,4 m+2, \ldots, 3 m+2,3 m+4) \text {, and } \\
& \mathbf{g}=(3 m+4,2 m+1,2,2 m, 3, \ldots, m+2, m+1) \text {. }
\end{aligned}
$$

LEMMA 2.14. There exists an ISOLS $(3 k+4 ; k)$ for $k$ odd, $k \geqslant 3$.

Proof. First we do the cases $k=3,5,15$. When $k=3$, put $d=1$, e $=$ $\left(1,5,10,9,3,2,8, x_{1}, x_{2}, x_{3}\right), \mathrm{f}=(4,6,7)$ and $\mathrm{g}=(10,3,5)$; and when $k=5$, put $d=1, \mathrm{e}=\left(1,4,6,8,12,5,3,2,14, x_{1}, x_{2}, x_{3}, x_{4}, x_{5}\right), \mathrm{f}=(7,9,10,11,13)$, and $\mathrm{g}$ $=(2,13,12,10,7)$. For $k=15$, use Lemma 1.6 with $p=5, q=9, x=3, a_{p 0}=1$ and $a_{11}=3$. (Note that product yields an $\operatorname{IPOLS}(12 ; 3,3)$, and that we have slightly varied the lemma by using an ISOLS $(13 ; 3)$.)

Now, write $k=3^{\alpha} 5^{\beta} K$, where $\operatorname{gcd}(K, 30)=1$, can assume that $k \neq 3,5,15$. There are four cases to consider, and, in each, Lemma 1.9 is applied with $a_{p 0}=a_{11}=a_{2}=1$. If both $\alpha \neq 1$ and $\beta \neq 1$, put $p=k, q=3$ and $x=1$; if $\alpha \neq 1$ but $\beta=1$, put $p=k / 5, q=15$ and $x=5$; if $\alpha=1$ but $\beta \neq 1$, put $p=k / 3, q=9$ and $x=3$; and if $\alpha=\beta=1$, put $p=k / 15, q=45$ and $x=15$. 
LEMMA 2.15. There exists an ISOLS $(3 k+7 ; k)$ when $k$ is odd and $k \geqslant 3$.

Proof. The proof is essentially the same as that of Lemma 2.14. We first construct ISOLS $(3 k+7 ; k)$ for $k=3,5$ and 15 and then proceed as before, applying Lemma 1.9 with $a_{p 0}=a_{2}=1$ and $A_{1}=\left\{1^{4}\right\}$. (Note that we also need Lemmas 2.13 and 2.14.)

An ISOLS $(16 ; 3)$ and an ISOLS $(22 ; 5)$ are given by $d=1, \mathbf{e}=(1,4$, $\left.x_{1}, 10,12,9,8,7,6,2, x_{2}, 3, x_{3}\right), \mathrm{f}=(5,11,13), \mathrm{g}=(9,12,10)$ and by $d=14, \mathrm{e}=$ $\left(1, x_{1}, x_{2}, x_{3}, x_{4}, 15, x_{5}, 7,3,16,12,8,4,17,13,9,5\right), \mathbf{f}=(2,6,10,11,14)$ and $\mathrm{g}=$ $(14.16,12,15,17)$, respectively. To construct an ISOLS(52;15), apply Lemma 1.6 (as in Lemma 2.14) with $p=5, q=9, x=3$ and $A_{0}=\left\{1,3^{2}\right\}$.

LEMMA 2.16. If $n=3 k+\varepsilon, 1 \leqslant \varepsilon<k, k \notin F \backslash\{3\}$ and if, when $k$ is even, $\varepsilon \neq 2,6$, then there is an $\operatorname{ISOLS}(3 k+\varepsilon ; k)$.

Proof. Suppose $k \geqslant 5$ and $k$ is odd. Then, for $\varepsilon \neq 3,4,7$, we apply Lemma 1.6 with $p=k, q=3, x=1, a_{p 0}=1$ and $A_{1}=\left\{1^{\varepsilon-1}\right\}$. If $k$ is even, $k \notin F$ and $\varepsilon \neq 2,3,6$, apply Lemma 1.6 with $p=k, q=3, x=1$ and $A_{1}=\left\{1^{\varepsilon}\right\}$ (with $a_{11}=1$ ).

All other cases in the statement of the lemma were dealt with in earlier lemmas.

LEMMA 2.17. If $k \in F \backslash\{2,3,6\}$, then there exists an ISOLS $(3 k+\varepsilon ; k), 1 \leqslant \varepsilon$ $<k$ and $\varepsilon \neq 2,6$.

Proof. We may assume that $\varepsilon \geqslant 4$, as the other cases have been dealt with. There are twenty-seven values of $k$ to consider.

For $k=10$, there are five cases: $n \in\{34,35,37,38,29\}$. When $n=35$, use Lemma 1.6 with $p=5, q=7$ and $x=2$; and for the others use Lemma 1.8 with $p=4$ and $q=8, a_{11}=2 ; q=9, a_{11}=1 ; q=7, A_{1}=\left\{3^{3}, 1\right\} ;$ and $q=8$, $A_{1}=\left\{2^{3}, 1\right\} ;$ respectively.

For $k \in G=\{14,46,54,58,62,74,82,98,118,142,194,202,214,278$, $394,398,422,1322\}, k / 2$ is an odd prime power. Apply Lemma 1.9 with $p=k / 2$, $q=6$ and $x=2$. If $\varepsilon \leqslant k-5$ is odd, then, if $\varepsilon \leqslant k / 2$, put $A_{0}=\left\{1^{\varepsilon}\right\}$; and if $\varepsilon>k / 2$, put $A_{0}=\left\{1^{k / 2}\right\}$ and $a_{2}=\cdots a_{b}=1$, where $b=\frac{1}{2}(\varepsilon-k / 2)+1$. If $\varepsilon$ is even and $\varepsilon \leqslant k-4$, then, if $\varepsilon \leqslant k / 2$, put $A_{0}=\left\{1^{\varepsilon^{-3}}\right\}, a_{11}=a_{2}=1$; and if $\varepsilon>k / 2$, put $A_{0}=\left\{1^{k / 2}\right\}, a_{11}=a_{2}=\cdots=a_{b}=1$ (if $\varepsilon=k / 2+1$ we just have $\left.a_{11}=1\right)$, where $b=\frac{1}{2}(\varepsilon-k / 2-1)+1$. 
For $k \in H=\{66,102,258,282,402\}, k / 3 \notin F$. In Lemma 1.6 put $p=k / 3$, $q=9, x=3$ and $A_{1}=\left\{1^{u}, 3^{v}\right\}$, where $1 \leqslant u+v \leqslant k / 3, a_{11}=1$ and $u+3 v=$ $\varepsilon$. Clearly $u$ and $v$ can always be found, provided that $\varepsilon \neq k-1, k-3$.

When $k \in G \cup H \backslash\{14,46,258,402\},(k+6) / 4 \notin F$ and $(k+6) / 4 \geqslant 7$. From the preceding lemmas there is an $\operatorname{ISOLS}(n ;(k+6) / 4)$ for $n \geqslant$ $3((k+6) / 4)+7$. Using Lemma 1.8 , put $p=4, q=3(k-2) / 4$, and $A_{1}=\left\{a_{11}=(k+6) / 4, a_{21}, a_{31}, a_{41}\right\}$, so that $0 \leqslant a_{j 1} \leqslant[3(k-2) / 8], j=2,3,4$, and $a_{21}+a_{31}+a_{41}=\varepsilon-((k+6) / 4) \geqslant 2((k+6) / 4)+7$; all of these are possible for $k \geqslant 58,54,50$ when $\varepsilon=k-3, k-2, k-1$, respectively. This leaves $k=54, \varepsilon=51$. In this case apply Lemma 1.5 with $p=5, q=31, A_{0}=$ $\left\{13^{2}, 14^{2}\right\}$ and $A_{1}=\left\{1^{4}\right\}$.

The cases $k=14,46$ and $\varepsilon=k-3, k-2, k-1$; and $k=258,402$ and $\varepsilon=k-3, k-1$ are shown in Table 2.4. This leaves only the cases $k=70,174$ and 230 .

TABLE 2.4

\begin{tabular}{rrrrllll}
$k$ & \multicolumn{1}{c}{$\boldsymbol{c}$} & \multicolumn{1}{c}{$\boldsymbol{p}$} & \multicolumn{1}{c}{$\boldsymbol{q}$} & \multicolumn{1}{c}{$\boldsymbol{A}_{\mathbf{0}}$} & \multicolumn{1}{c}{$\boldsymbol{A}_{\mathbf{1}}$} & $a_{2}$ Lemma & \\
\hline 14 & 11 & 7 & $\mathbf{6}$ & $\left\{1^{5}\right\}$ & $\left\{1^{4}\right\}$ & 1 & $1.9 x=2$ \\
14 & 12 & 8 & 5 & - & $\left\{2^{7}\right\}$ & - & 1.5 \\
14 & 13 & 7 & 6 & $\left\{1^{7}\right\}$ & $\left\{1^{4}\right\}$ & 1 & $1.9 x=2$ \\
$\mathbf{4 6}$ & 43 & 5 & 27 & $\left\{11^{2}, 12^{2}\right\}$ & - & - & 1.5 \\
$\mathbf{4 6}$ & 44 & 5 & 27 & $\left\{11^{2}, 12^{2}\right\}$ & $\{1\}$ & - & 1.5 \\
46 & 45 & 7 & 19 & $\left\{7^{2}, 8^{4}\right\}$ & $\left\{1^{4}\right\}$ & - & 1.5 \\
258 & 255 & 77 & 10 & $\left\{1^{2}, 2^{3}, 5^{50}\right\}$ & $\{1\}$ & - & 1.5 \\
258 & 257 & 45 & 17 & $\left\{2,8^{32}\right\}$ & $\left\{1^{8}\right\}$ & - & 1.5 \\
402 & 399 & 149 & 8 & $\left\{2,4^{100}\right\}$ & $\left\{1^{11}\right\}$ & - & 1.5 \\
402 & 401 & 149 & 8 & $\left\{2,4^{100}\right\}$ & $\left\{1^{13}\right\}$ & - & 1.5
\end{tabular}

We first look at $k=174,230$. Since $k \notin E$, there are at least $k / 2$ disjoint symmetric transversals in $P$, determined by $P_{1}$, all of which avoid the main diagonal. Use these in Lemma 1.6 with $p=k, q=3, x=1$ and $A_{1}=\left\{1^{\varepsilon}\right\}$ for $4 \leqslant \varepsilon \leqslant k / 2+1$. (Note that we also use the main diagonal of $P$, which is a transversal.)

For $k=174$ and $89 \leqslant \varepsilon \leqslant 169$, write $3 k+\varepsilon=29 \cdot 21+(\varepsilon-87)$, where $2 \leqslant$ $\varepsilon-87 \leqslant 82$, and then in Lemma 1.9 put $p=29, q=21, x=6, A_{0}=\left\{1^{t}\right\}$, $A_{1}=\left\{1^{s}\right\}$ and $a_{2}=\cdots=a_{u}=1$, so that $0 \leqslant s, t \leqslant 29, s, t \neq 2,3,6, u \leqslant 13$ and $2 \leqslant t+s+2(u-1) \leqslant 82$. (Any ISOLS $(n ; 6)$ required which have not already been constructed are given in Lemma 2.22). For the remaining four cases, $170 \leqslant \varepsilon \leqslant 173$, use Lemma 1.8 with $p=4, q=117$ and $A_{1}=\left\{a_{11}=\right.$ $\left.57, a_{12}, a_{13}, a_{14}\right\}$ where $171 \geqslant a_{12}+a_{13}+a_{14}=\varepsilon+54-57=\varepsilon-3 \geqslant 115$.

The case $k=230$ is handled similarly. When $117 \leqslant \varepsilon \leqslant 156$, write $3 k+\varepsilon=$ $23 \cdot 35+(\varepsilon-115)$, and in Lemma 1.9 put $p=23, q=35, x=10, A_{0}=\left\{1^{t}\right\}$, $A_{1}=\left\{1^{s}\right\}, a_{2}=\cdots=a_{u}=1$, so that $t \neq 2,6$ is even, $0 \leqslant t \leqslant 22, s=0$ or 1 , 
TABLE 2.5

\begin{tabular}{|c|c|c|c|c|c|}
\hline$p$ & $q$ & $\boldsymbol{\varepsilon}$ & $A_{0}$ & $A_{1}$ & $a_{2}$ \\
\hline 7 & 30 & 4 & $\{1\}$ & $\{1\}$ & 1 \\
\hline 7 & 30 & 5 & $\left\{1^{5}\right\}$ & - & - \\
\hline 7 & 30 & 7 & $\left\{1^{7}\right\}$ & - & - \\
\hline 7 & 30 & 8 & $\left\{1^{5}\right\}$ & $\{1\}$ & 1 \\
\hline 7 & 30 & 9 & $\left\{1^{7}\right\}$ & - & 1 \\
\hline 7 & 30 & 10 & $\left\{1^{7}\right\}$ & $\{1\}$ & 1 \\
\hline 7 & 30 & 11 & $\left\{1^{4}\right\}$ & $\left\{1^{7}\right\}$ & - \\
\hline 7 & 30 & 12 & $\left\{10,1^{2}\right\}$ & - & - \\
\hline 7 & 30 & 13 & $\left\{1^{4}\right\}$ & $\left\{1^{7}\right\}$ & - \\
\hline 7 & 31 & 14 & $\left\{1^{4}\right\}$ & \{1\} & 1 \\
\hline 7 & 32 & 15 & $\{1\}$ & - & - \\
\hline 7 & 32 & 16 & $\{1\}$ & $\{1\}$ & - \\
\hline 7 & 32 & 17 & $\{1\}$ & - & 1 \\
\hline 7 & 32 & 19 & $\{1\}$ & $\left\{1^{4}\right\}$ & - \\
\hline 7 & 32 & 20 & $\{1\}$ & $\left\{1^{5}\right\}$ & - \\
\hline 7 & 32 & 21 & $\{1\}$ & $\left\{1^{4}\right\}$ & 1 \\
\hline 7 & 32 & 22 & $\{1\}$ & $\left\{1^{5}\right\}$ & 1 \\
\hline 7 & 32 & 23 & $\left\{1^{5}\right\}$ & $\left\{1^{4}\right\}$ & - \\
\hline 7 & 32 & 24 & $\left\{1^{5}\right\}$ & $\left\{1^{5}\right\}$ & - \\
\hline 7 & 32 & 25 & $\left\{1^{5}\right\}$ & $\left\{1^{4}\right\}$ & 1 \\
\hline 7 & 32 & 26 & $\left\{1^{5}\right\}$ & $\left\{1^{5}\right\}$ & 1 \\
\hline 7 & 33 & 27 & $\{1\}$ & $\left\{1^{5}\right\}$ & - \\
\hline 7 & 33 & 28 & $\left\{1^{7}\right\}$ & - & - \\
\hline 7 & 33 & 29 & $\left\{1^{4}\right\}$ & $\left\{1^{4}\right\}$ & - \\
\hline 7 & 33 & 30 & $\left\{1^{5}\right\}$ & $\left\{1^{4}\right\}$ & - \\
\hline 7 & 33 & 31 & $\left\{1^{5}\right\}$ & $\left(1^{5}\right\}$ & - \\
\hline 7 & 34 & 32 & - & $\left\{1^{4}\right\}$ & - \\
\hline 7 & 34 & 33 & - & $\left\{1^{5}\right\}$ & - \\
\hline 7 & 34 & 34 & - & $\left\{1^{4}\right\}$ & 1 \\
\hline 7 & 34 & 35 & $\left\{1^{5}\right\}$ & - & 1 \\
\hline 7 & 34 & 36 & $\left\{1^{5}\right\}$ & $\{1\}$ & 1 \\
\hline 7 & 34 & 37 & - & - & 1 \\
\hline 7 & 35 & 38 & - & $\{1\}$ & 1 \\
\hline 7 & 35 & 39 & $\left\{1^{4}\right\}$ & - & - \\
\hline 7 & 35 & 41 & $\left\{1^{4}\right\}$ & - & 1 \\
\hline 7 & 35 & 42 & $\left\{1^{4}\right\}$ & $\{1\}$ & 1 \\
\hline 7 & 36 & 43 & $\{1\}$ & - & - \\
\hline 7 & 36 & 44 & $\{1\}$ & $\{1\}$ & - \\
\hline 7 & 36 & 45 & $\{1\}$ & - & 1 \\
\hline 7 & 36 & 46 & $\{1\}$ & $\{1\}$ & 1 \\
\hline 7 & 36 & 47 & $\left\{1^{5}\right\}$ & - & - \\
\hline
\end{tabular}

and, if $s=1$, then at least one $a_{j}$ is non-zero, and $u \leqslant 10$. It follows that $2 \leqslant s+t+2(u-1) \leqslant 41$. (All the restrictions on $s$ and $t$ are to ensure that an ISOLS $(36 ; 10)$ is not required.) For $157 \leqslant \varepsilon \leqslant 229$, we use Lemma 1.8 with $p=4, q=155$ and $A_{1}=\left\{a_{11}=75, a_{12}, a_{13}, a_{14}\right\}$, where $231 \geqslant a_{12}+a_{13}+a_{14}$ $=\varepsilon+70-75=\varepsilon-5 \geqslant 152$.

This leaves only the case $k=70$. The values given in Table 2.5 used in Lemma 1.6 or 1.9 with $x=10$ cover all cases $\varepsilon \leqslant 47$ except $\varepsilon=18$, when we set $p=5$, 
$q=42, x=14$ and $A_{0}=\left\{14,1^{4}\right\}$ in Lemma 1.6 , and $\varepsilon=40$, when we put $p=20, q=9$ and $A_{1}=\left\{4^{17}, 1^{2}\right\}$ in Lemma 1.5. When $48 \leqslant \varepsilon \leqslant 70$, use Lemma 1.8 with $p=4, q=47$ and $A_{1}=\left\{a_{11}=23, a_{12}, a_{13}, a_{14}\right\}$, where $69 \geqslant a_{12}+a_{13}$ $+a_{14}=\varepsilon+22-23=\varepsilon-1 \geqslant 47$.

THEOREM 2.18. If $n=3 k+\varepsilon, 1 \leqslant \varepsilon<k$ and $\varepsilon \neq 2,6$ if $k$ is even, then there exists an ISOLS( $n ; k)$.

Proof. Combine the last six lemmas.

Although many infinite families of ISOLS $(6 m+6 ; 2 m)$ can be constructed, we know of no ISOLS $(6 m+2 ; 2 m)$ and computer search verifies that the smallest of these, an ISOLS $(8 ; 2)$, does not exist.

In order to complete our work it remains only to construct ISOLS $(n ; k)$, $n \geqslant 3 k+1$ and $3 \leqslant k \leqslant 6$. It follows from the existence of $\operatorname{ISOLS}(n ; m)$ for $n \geqslant 3 m+1$ when $m$ is odd, and for $n \geqslant 3 m+7$ when $m$ is even, and of an $\operatorname{ISOLS}(3 k+1 ; k), k \in\{3,4,5,6\}$, that an $\operatorname{ISOLS}(n ; k), k \in\{3,5\}$, exists for all $n \geqslant 9 k+10$, and that ISOLS $(n ; k), k \in\{4,6\}$, exist for all $n \geqslant 9 k+4$. Many of the small orders have been constructed by other authors (for example Bennett [1], Bennett and Mendelsohn [2], Drake and Larson [8] and Wang [19]).

THEOREM 2.19. There exists an ISOLS( $n ; 3)$ for all $n \geqslant 10$.

Proof. We have only to consider integers $n \in\{m: 14 \leqslant m \leqslant 37\} \backslash\{16\}$. First, the cases $n \in\{14,17,20,21,22,26,32\}$ are given by $d$ and by the vectors $e, f$ and $g$ in Table 2.6. An ISOLS(33; 3$)$ exists as there exists an ISOLS(33; 10). Applying Lemma 1.5 with $q=3$, and with the other variables as in Table 2.7, we obtain the remainder.

THEOREM 2.20. There exists an ISOLS $(n ; 4)$ for all $n \geqslant 13$, except perhaps for $n=14$.

Proof. Only $n$ in the range $16 \leqslant n \leqslant 39$ need be considered. If $n=4 t, t \neq 6$, product gives the result, and if $n=4 t+1, t \neq 6$, we use Lemma 1.8 with $p=t$, $q=4$ and $A_{1}=\{1\}\left(a_{11}=0\right)$. The cases $n=18,26,30$ were found by Wang [19] and are constructed using the starter-added technique, as also are $n=19,23,27$ (see Table 2.8). The remaining constructions are given in Table 2.9.

THEOREM 2.21. There exists an ISOLS( $n ; 5)$ for all $n \geqslant 16$. 
TABLE 2.6

\begin{tabular}{rrlrl}
$n$ & $d$ & $\mathrm{e}$ & \multicolumn{1}{c}{$\mathbf{f}$} & $\mathrm{g}$ \\
14 & 9 & $\left(1, x_{1}, x_{2}, 3,11, x_{3}, 5,2,10,7,4\right)$ & $(6,8,9)$ & $(10,11,7)$ \\
17 & 1 & $\left(1,3,5,8, x_{1}, 13,12,7, x_{2}, x_{3}, 6,9,2,10\right)$ & $(4,11,14)$ & $(7,13,9)$ \\
20 & 14 & $\left(1, x_{1}, x_{2}, 6,2,15,11,7,3,16,12,8, x_{3}, 17,13,9,5\right)$ & $(4,10,14)$ & $(16,17,6)$ \\
21 & 1 & $\left(1, x_{1}, x_{2}, x_{3}, 2,4,8,13,16,9,15,7,6,5,3,18,11,10\right)$ & $(12,14,17)$ & $(9,4,15)$ \\
22 & 5 & $\left(1, x_{1}, x_{2}, 13,17,2,6,10, x_{3}, 18,3,7,11,15,19,4,8,12,16\right)$ & $(5,9,14)$ & $(18,12,19)$ \\
26 & 1 & $\left(1, x_{1}, x_{2}, x_{3}, 2,4,6,9,12,18,22,16,15,21,20,8,10,7,5,3,11,17,14\right)$ & $(13,19,23)$ & $(11,18,20)$ \\
32 & 1 & $\left(1, x_{1}, x_{2}, x_{3}, 24,6,9,11,13,15,26,8,21,28,27,25,23,29,16,14\right.$, & $(18,20,24)$ & $(19,16,21)$ \\
& & $12,10,7,5,3,19,22,7)$ & &
\end{tabular}

TABLE 2.7

\begin{tabular}{rrll}
$n$ & $p$ & $A_{0}$ & $A_{1}$ \\
\hline 15 & 4 & - & $\left\{1^{3}\right\}\left(a_{11}=1\right)$ \\
18 & 5 & $\left\{1^{3}\right\}$ & - \\
19 & 5 & $\left\{1^{3}\right\}$ & $\{1\}$ \\
23 & 5 & $\left\{1^{3}\right\}$ & $\left\{1^{5}\right\}$ \\
24 & 7 & $\left\{1^{3}\right\}$ & - \\
25 & 7 & $\left\{1^{3}\right\}$ & $\{1\}$ \\
27 & 8 & - & $\left\{1^{3}\right\}\left(a_{11}=1\right)$ \\
28 & 7 & $\left\{1^{3}\right\}$ & $\left\{1^{4}\right\}$ \\
29 & 7 & $\left\{1^{3}\right\}$ & $\left\{1^{5}\right\}$ \\
30 & 9 & $\left\{1^{3}\right\}$ & - \\
31 & 9 & $\left\{1^{3}\right\}$ & $\{1\}$ \\
34 & 9 & $\left\{1^{3}\right\}$ & $\left\{1^{4}\right\}$ \\
35 & 9 & $\left\{1^{3}\right\}$ & $\left\{1^{5}\right\}$ \\
36 & 11 & $\left\{1^{3}\right\}$ & - \\
37 & 11 & $\left\{1^{3}\right\}$ & $\{1\}$
\end{tabular}

TABLE 2.8

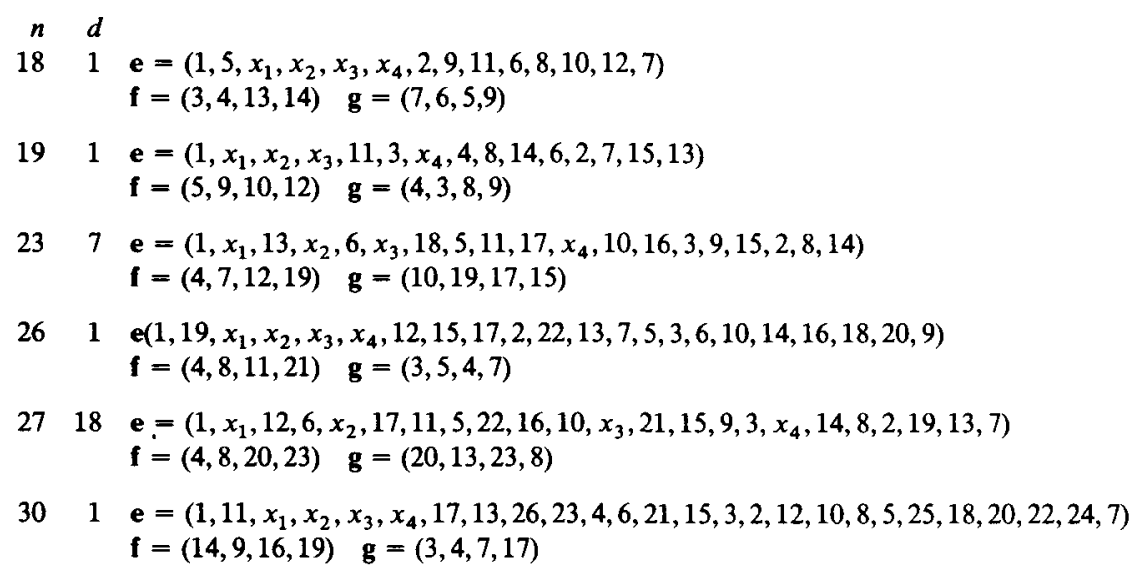


TABLE 2.9

\begin{tabular}{rrrlll}
$n$ & $p$ & $q$ & $A_{0}$ & $A_{1}$ & Lemma \\
\hline 22 & 7 & 3 & $\{1\}$ & - & 1.8 \\
24 & 5 & 4 & $\left\{1^{4}\right\}$ & - & 1.5 \\
25 & 5 & 4 & $\left\{2^{2}\right\}$ & $\{1\}$ & 1.5 \\
31 & 10 & 3 & $\{1\}$ & - & 1.8 \\
34 & 11 & 3 & $\{1\}$ & - & 1.8 \\
35 & 4 & 7 & - & $\left\{1,3^{2}\right\}$ & $1.6 x=1$ \\
38 & 11 & 3 & $\{1\}$ & $\left\{1^{4}\right\}$ & 1.5 \\
39 & 5 & 7 & $\left\{1^{4}\right\}$ & - & 1.5
\end{tabular}

TABLE 2.10

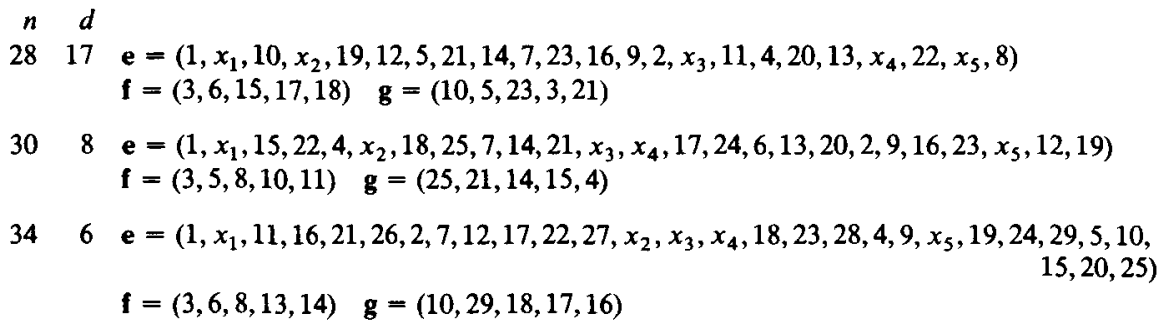

TABLE 2.11

\begin{tabular}{rrrlll}
$n$ & $p$ & $q$ & $A_{0}$ & $A_{1}$ & Lemma \\
\hline 23 & 4 & 4 & - & $\left\{1,2^{3}\right\}$ & 1.8 \\
24 & 4 & 5 & - & $\left\{2^{2}\right\}$ & 1.8 \\
26 & 7 & 3 & $\left\{1^{5}\right\}$ & - & 1.5 \\
27 & 5 & 5 & - & $\{2\}$ & 1.8 \\
29 & 7 & 4 & $\{1\}$ & - & 1.8 \\
31 & 7 & 4 & $\{1\}$ & $\{2\}$ & 1.8 \\
32 & 9 & 3 & $\left\{1^{5}\right\}$ & - & 1.5 \\
33 & 8 & 4 & - & $\{1\}$ & 1,8 \\
37 & 5 & 7 & - & $\{2\}$ & $1.6 x=1$ \\
38 & 5 & 7 & $\{1\}$ & $\{2\}$ & $1.6 x=1$ \\
39 & 5 & 7 & $\left\{2^{2}\right\}$ & - & $1.6 x=1$ \\
42 & 5 & 8 & - & $\{2\}$ & $1.6 x=1$ \\
43 & 5 & 8 & - & $\{3\}$ & $1.6 x=1$ \\
44 & 5 & 8 & $\left\{2^{2}\right\}$ & - & $1.6 x=1$ \\
47 & 5 & 9 & - & $\{2\}$ & $1.6 x=1$ \\
48 & 5 & 9 & - & $\{3\}$ & $1.6 x=1$ \\
49 & 5 & 9 & - & $\{4\}$ & $1.6 x=1$ \\
52 & 5 & 10 & $\{1\}$ & $\{1\}$ & $1.6 x=1$ \\
53 & 5 & 10 & - & $\{3\}$ & $1.6 x=1$ \\
54 & 5 & 10 & $\left\{2^{2}\right\}$ & - & $1.6 x=1$
\end{tabular}


TABLE 2.12

\begin{tabular}{|c|c|c|c|c|c|c|}
\hline$n$ & $p$ & $q$ & $A_{0}$ & $A_{1}$ & $a_{2}$ & Lemma \\
\hline 22 & 4 & 4 & - & $\left\{2^{3}\right\}$ & - & 1.5 \\
\hline 24 & 7 & 3 & $\{1\}$ & - & 1 & 1.8 \\
\hline 25 & 7 & 3 & $\{1\}$ & $\{1\}$ & 1 & 1.8 \\
\hline 26 & 4 & 5 & - & $\left\{2^{3}\right\}$ & - & 1.5 \\
\hline 27 & 5 & 4 & $\{1\}$ & $\left\{2^{3}\right\}$ & - & 1.5 \\
\hline 28 & 7 & 3 & $\{1\}$ & $\left\{1^{6}\right\}$ & - & 1.5 \\
\hline 29 & 7 & 3 & $\{1\}$ & $\left\{1^{5}\right\}$ & 1 & 1.8 \\
\hline 30 & 8 & 3 & - & $\left\{1^{6}\right\}$ & - & 1.5 \\
\hline 31 & 5 & 5 & $\left\{2^{3}\right\}$ & - & - & 1.5 \\
\hline 32 & 7 & 3 & $\left\{1^{5}\right\}$ & $\left\{1^{6}\right\}$ & - & 1.5 \\
\hline 34 & 4 & 7 & - & $\left\{2^{3}\right\}$ & - & 1.5 \\
\hline 35 & 7 & 4 & $\{1\}$ & $\left\{2^{3}\right\}$ & - & 1.5 \\
\hline 36 & 11 & 3 & $\{1\}$ & - & 1 & 1.8 \\
\hline 37 & 11 & 3 & $\{1\}$ & $\{1\}$ & 1 & 1.8 \\
\hline 38 & 4 & 8 & - & $\left\{2^{3}\right\}$ & - & 1.5 \\
\hline 39 & 7 & 4 & $\left\{1^{5}\right\}$ & $\left\{2^{3}\right\}$ & - & 1.5 \\
\hline 40 & 9 & 3 & $\left\{1^{7}\right\}$ & $\left\{1^{6}\right\}$ & - & 1.5 \\
\hline 41 & 7 & 5 & $\left\{2^{3}\right\}$ & - & - & 1.5 \\
\hline 42 & 13 & 3 & $\{1\}$ & - & 1 & 1.8 \\
\hline 43 & 13 & 3 & $\{1\}$ & $\{1\}$ & 1 & 1.8 \\
\hline 44 & 11 & 3 & $\left\{1^{5}\right\}$ & $\left\{1^{6}\right\}$ & - & 1.5 \\
\hline 45 & 5 & 7 & $\left\{1^{4}\right\}$ & $\left\{2^{3}\right\}$ & - & 1.5 \\
\hline 46 & 4 & 10 & - & $\left\{2^{3}\right\}$ & - & 1.5 \\
\hline 47 & 9 & 4 & $\left\{1^{5}\right\}$ & $\left\{2^{3}\right\}$ & - & 1.5 \\
\hline 48 & 11 & 3 & $\left\{1^{9}\right\}$ & $\left\{1^{6}\right\}$ & - & 1.5 \\
\hline 49 & 9 & 4 & $\left\{1^{7}\right\}$ & $\left\{2^{3}\right\}$ & - & 1.5 \\
\hline 50 & 11 & 4 & $\left\{1^{6}\right\}$ & - & - & 1.5 \\
\hline 51 & 11 & 4 & $\{1\}$ & $\left\{2^{3}\right\}$ & - & 1.5 \\
\hline 52 & 5 & 9 & $\{1\}$ & $\left\{2^{3}\right\}$ & - & 1.5 \\
\hline 53 & 13 & 3 & $\left\{1^{11}\right\}$ & $\{1\}$ & 1 & 1.8 \\
\hline 54 & 4 & 12 & - & $\left\{2^{3}\right\}$ & - & 1.5 \\
\hline 55 & 7 & 7 & $\left\{2^{3}\right\}$ & - & - & 1.5 \\
\hline 56 & 5 & 10 & $\left\{2^{3}\right\}$ & - & - & 1.5 \\
\hline 57 & 5 & 10 & $\{1\}$ & $\left\{2^{3}\right\}$ & - & 1.5 \\
\hline
\end{tabular}

Proof. As before we need only consider $n$ in the range $20 \leqslant n \leqslant 54$, but with $n \neq 5 t$ or $5 t+1$ unless $n=26,30,31$, as in the first case, product suffices, and, in the second, Lemma 1.6 applies with $p=5, q=t, x=1$ and $A_{0}=\{1\}$. The cases $n=28,30,34$ are constructed from vectors as in Table 2.10, and the remainder are given in Table 2.11. Recall that an ISOLS $(22 ; 5)$ was given in Lemma 2.15.

ThEOREM 2.22. There exists an $\operatorname{ISOLS}(n ; 6)$ for all $n \geqslant 19$, except perhaps for $n=20$. 
Proof. For $n=19$ and 21 , ISOLS $(n ; 6)$ have been given. Only two, $n=23$ and $n=33$ are constructed via the "starter-adder" method. For $n=23$, put $d=15, \mathrm{e}=\left(1, x_{1}, x_{2}, x_{3}, x_{4}, x_{5}, 17,14, x_{6}, 8,5,2,16,13,10,7,4\right), \quad \mathrm{f}=$ $(3,6,9,11,12,15)$ and $\mathrm{g}=(16,15,10,14,17,13)$; and for $n=33$, put $d=1$, $\mathbf{e}=\left(1, x_{1}, x_{2}, x_{3}, x_{4}, x_{5}, x_{6}, 2,4,6,8,10,12,20,22,21,19,9,7,5,3,25,27,11,18\right.$, $15,13), \mathbf{f}=(14,16,17,23,24,26)$ and $g=(12,11,18,9,20,2)$. The remainder are given in Table 2.12 .

\section{Acknowledgements}

The first author acknowledges the financial support of the National Sciences and Engineering Research Council of Canada under grant A-5075, and the hospitality of the University of Newcastle during Spring 1984. We also thank Brenda Li of the Computing Science Department at Simon Fraser University for the computer results.

\section{References}

[1] F. E. Bennett, Self-orthogonal latin squares with self-orthogonal subsquares, unpublished manuscript.

[2] F. E. Bennett and N. S. Mendelsohn, 'On the spectrum of Stein quasigroups', Bull. Austral. Math. Soc. 21 (1980), 47-63.

[3] R. B. Brayton, D. Coppersmith and A. J. Hoffman, Self-orthogonal latin squares of all orders $n \neq 2,3,6$ (Coll. Internationale sulle Teorie Combinatorie, Roma, 1973, Atti dei convegni Lincei, No. 17, Tomo II, 1976), pp. 509-517.

[4] A. E. Brouwer and G. H. J. van Rees, 'More mutually orthogonal latin squares', Discrete Math. 39 (1982), 263-281.

[5] D. J. Crampin and A. J. W. Hilton, 'On the spectra of certain types of latin square', $J$. Combinatorial Theory 19 (1975), 84-94.

[6] J. Dénes and A. D. Keedwell, Latin squares and their applications (Akadémiai Kiadó, Budapest, 1974).

[7] J. H. Dinitz and D. R. Stinson, 'Mols with holes', Discrete Math. 44 (1983), 145-154.

[8] D. A. Drake and J. A. Larson, 'Pairwise balanced designs whose line sizes do not divide six', $J$. Combin. Theory Ser. A 34 (1983), 266-300.

[9] D. A. Drake and H. Lenz, 'Orthogonal latin squares with orthogonal subsquares', Archiv der Mathematik 34 (1980), 565-576.

[10] A. Hedayat, 'A generalization of sum composition: self-orthogonal latin square design with sub self orthogonal latin square designs', J. Combin. Theory Ser. A 24 (1978), 202-210.

[11] A. Hedayat and E. Seiden, 'On the theory and application of sum composition of latin squares and orthogonal latin squares', Pacific J. Math. 54 (1974), 85-113.

[12] K. Heinrich, 'Self-orthogonal latin squares with self-orthogonal subsquares', Ars Combinatoria 3 (1977), 251-266.

[13] K. Heinrich, Latin squares with and without subsquares of prescribed type, Ann. Discrete Math., to appear. 
[14] K. Heinrich and L. Zhu, 'Existence of orthogonal latin squares with aligned subsquares', Discrete Math. 59 (1986), 69-78.

[15] J. D. Horton, 'Sub-latin squares and incomplete orthogonal arrays', J. Combin. Theory 16 (1974), 23-33.

[16] C. C. Lindner, R. C. Mullin and D. R. Stinson, 'On the spectrum of resolvable orthogonal arrays invariant under the Klein group $K_{4}$ ', Aequationes Math. 26 (1983), 176-183.

[17] E. T. Parker, 'Orthogonal latin squares', Proc. Nat. Acad. Sci. U. S. A. 45 (1959), 859-862.

[18] W. D. Wallis and L. Zhu, Orthogonal latin squares with small subsquares (Combinatorial Mathematics X (Adelaide 1982), Lecture Notes in Math., Vol. 1036, Springer-Verlag, Berlin and New York, 1983), pp. 398-409.

[19] S. M. P. Wang, On self-orthogonal latin squares and partial transversals of latin squares $(\mathrm{Ph} . \mathrm{D}$. thesis, Ohio State University, Columbus, Ohio, 1978).

[20] L. Zhu, 'A short disproof of Euler's conjecture concerning orthogonal latin squares', Ars Combinatoria 14 (1982), 47-55.

[21] L. Zhu, 'Some results on orthogonal latin squares with orthogonal subsquares', Utilitas Math. 25 (1984), 241-248.

[22] L. Zhu, 'Orthogonal latin squares with subsquares', Discrete Math. 48 (1984), 315-321.

[23] L. Zhu, 'A few more self-orthogonal latin squares with symmetric orthogonal mates', Congr. Numer. 42 (1984), 313-320.

Department of Mathematics

Simon Fraser University

Burnaby, B. C., V5A 1SB

Canada
Department of Mathematics

Suzhou University

Suzhou

People's Republic of China 